\title{
Caractérisation paysanne de Hippopotamus amphibius Linné 1758, dans la Réserve de Biosphère de la Mare aux Hippopotames, en zone sud soudanienne du Burkina Faso
}

\author{
Ollo Théophile DIBLONI ${ }^{1 *}$, Nessan Désiré COULIBALY ${ }^{1}$, Wendengoudi GUENDA ${ }^{2}$, \\ Cédric VERMEULEN ${ }^{3}$ et Mamounata BELEM/OUEDRAOGO ${ }^{1}$ \\ ${ }^{I}$ INERA/DPF 03 BP 7047 Ouagadougou 03, Burkina Faso. \\ ${ }^{2}$ UFR/SVT, Université de Ouagadougou 03 BP 7021, Burkina Faso. \\ ${ }^{3}$ Unité GRFMN, Laboratoire de Foresterie des régions tropicales et subtropicales/FUSAGX, \\ Passage des Déportés 2, 5030 Gembloux, Belgique. \\ *Auteur correspondant, Email : dibloni@yahoo.fr, Tel. (+226) 503340 98, Fax: (+226) 50315003
}

\section{RESUME}

L'étude de la caractérisation de l'hippopotame par les populations villageoises a visé à inventorier les savoirs endogènes sur l'espèce dans la Réserve de Biosphère de la Mare aux Hippopotames du Burkina Faso. L'inventaire de ces savoirs a porté sur l'effectif des populations d'hippopotames, la différenciation sexuelle, le régime alimentaire, les valeurs socioéconomiques et culturelles ainsi que les dégâts dus à cette espèce. L'enquête a été conduite dans six villages de l'ethnie Bobo et a concerné un échantillon de 77 personnes. Les personnes enquêtées savent que les hippopotames sont présents dans la réserve et estiment leur effectif à environ 33 individus. A l'aide de critères morphologiques et éthologiques, les villageois opèrent une différenciation sexuelle chez ces animaux. Le régime alimentaire est connu et comprendrait selon eux une quarantaine d'espèces végétales. Outre les valeurs socioéconomiques, culturelles et touristiques que la présence des hippopotames procure, les populations savent aussi que ces animaux participent au maintien de la biodiversité et à la fertilisation de la mare pour la production de poissons. Toutefois, l'espèce serait également à l'origine de la destruction des filets de pêche, de dégâts sur les cultures céréalières et de quelques accidents mortels.

(C) 2009 International Formulae Group. All rights reserved.

Mots clés : Caractérisation paysanne, Mare aux Hippopotames, Réserve de Biosphère, zone sud soudanienne, Burkina Faso.

\section{INTRODUCTION}

Le Burkina Faso, comme tous les pays soudano-sahéliens, a connu depuis 1970 des sécheresses récurrentes qui ont contribué considérablement à la dégradation de ses ressources naturelles. La persistance de ces aléas climatiques a entraîné les migrations des populations des zones dégradées au Nord vers les zones fertiles et plus arrosées au Sud du pays (Gomgnimbou et Bonou, 1996; Vermeulen, 2001; CICRED, 2006). Les villages de la Réserve de Biosphère de la Mare aux Hippopotames (RBMH) ont été une zone d'accueil pour ces nombreux migrants venus des zones défavorisées du Nord. Les environs immédiats de la Réserve sont emblavés de spéculations céréalières et cotonnières (UCF/Houet, 2006). Les agropasteurs s'incrustent souvent dans la Réserve pour l'exploitation de bois et des prélèvements de gibiers aux moyens de pièges, de chiens de chasse ou même d'armes à feu. Ces actions pourraient contribuer à la disparition des habitats de la faune déjà fragilisés par la présence du bétail et les coupes de bois pour l'artisanat ou l'ébénisterie 
(UCF/Houet, 2006; Kièma, 2007). Selon plusieurs auteurs (Noirard et al., 2004; Okoumasou et al., 2004; Binot et al., 2006), les périphéries proches des aires de conservation font l'objet de beaucoup de convoitises dans les zones arides et semiarides d'Afrique. Il ressort que ces périphéries constituent le plus souvent des zones de conflits entre les hommes et la faune sauvage (Hukol, 2000; Sam et al., 2002; Okoumasou et al., 2004; Binot et al., 2006). Dans ces situations, Binot et al. (2006) rapportent qu'en Afrique australe, les actions de protection de la faune sauvage ont plus de chance d'être efficaces lorsqu'elles attribuent à la faune sauvage une valeur économique. Pour ce faire, le développement et la promotion des revenus alternatifs issus de la faune doivent contribuer à sa gestion conservatrice ou durable. Ainsi donc, pour amener les populations villageoises à mieux préserver les ressources de la réserve, le développement des activités éco-touristiques constitue un des atouts susceptibles de créer des emplois pour les communautés locales vivant en bordure. Pour cela, il est utile de s'assurer des connaissances que ces populations villageoises ont de la réserve et surtout des rôles socio-économiques et culturels des hippopotames.

Les connaissances qu'ont les populations riveraines de l'hippopotame méritent d'être évaluées dans la perspective de leur plus grande implication dans la gestion de la réserve. C'est pourquoi l'objectif principal de cette étude a consisté à inventorier et à évaluer les savoirs endogènes locaux sur cette espèce.

\section{MATERIEL ET METHODES Site d'étude}

L'actuelle Réserve de Biosphère de la Mare aux Hippopotames (RBMH) était une forêt classée par arrêté n ${ }^{\circ} 8336$ SE du 26 mars 1937 qui a été intégrée au réseau des Réserves de Biosphère le 12 janvier 1987 par l'U.N.E.S.C.O. (Chardonnet, 1995; Poda, 1997; Taïta, 1997). Elle couvre une superficie approximative de 19200 hectares et est située à environ $60 \mathrm{~km}$ au nord de Bobo Dioulasso entre les latitudes $11^{\circ} 30^{\prime}$ et $11^{\circ} 45^{\prime}$ nord et les longitudes $04^{\circ} 05$ et $04^{\circ} 12$ ouest (carte 1 ). Son climat est de type soudanien avec des précipitations annuelles de l'ordre de 1100 $\mathrm{mm}$, réparties sur 4 à 6 mois, de mai à octobre
(Bélem, 2008). La végétation est constituée de plusieurs formations parmi lesquelles on distingue une végétation aquatique autour de la Mare, des forêts galeries, des forêts claires et des forêts denses sèches ainsi que des savanes arborées et arbustives (Taïta, 1997; Belem, 2008). La faune de cette réserve est célèbre pour ses hippopotames (Hippopotamus amphibius L.) qui y vivent en permanence et qui ont donné leur nom au lieu : «Réserve de Biosphère de la Mare aux Hippopotames ». D'autres mammifères sont également présents dans la Réserve (ENGREF, 1989; Bouché, 2005). Il s'agit de: éléphants (Loxodonta africana Cuvier), guibs harnachés (Tragelaphus scriptus Pallas), hippotragues (Hippotragus equinus Desmarest), phacochères (Phacocoerus africanus), ourébis (Ourebia ourebi Zimmerman), céphalophes (Cephalophus sp), waterbuck (Kobus ellipsiprymnus Ogilby), patas (Erythrocebus patas Schreber) et de babouins (Papio Papio doguera L), etc. L'avifaune est riche et variée (Poussy et Bakyono, 1991). Des pêcheurs installés en bordure de la Mare assurent l'exploitation halieutique ainsi que l'exploitation touristique du site en emmenant les visiteurs voir les hippopotames.

Les principaux problèmes de la réserve sont les feux tardifs, le braconnage pratiqué aussi bien à l'aide d'armes à feu, de pièges qu'à l'aide de chiens de chasse, les conflits hippopotames/pêcheurs (destruction des filets) et hippopotames/agriculteurs (ravage des champs). On compte aussi la pêche avec des engins prohibés, les pâturages illicites par les pasteurs transhumants et l'exploitation illicite de bois verts (UCF/Haut Bassin, 2004). Pour contrộler ces actions nocives à la durabilité de la Réserve, le Projet de partenariat pour la Gestion des Ecosystèmes Naturels (PAGEN) du Ministère de l'Environnement et du Cadre de Vie (MECV) et le Projet GEF/MABUNESCO ont mis en place l'Association villageoise pour la Gestion des Ressources Naturelles et de la Faune (AGEREF) qui est une structure faîtière communautaire réunissant les organisations des producteurs oeuvrant dans la zone sous influence de la réserve.

\section{Description de l'hippopotame commun \\ L'hippopotame commun (Hippopota- mus amphibius, Linné 1758) est un gros}


mammifère typiquement africain (Haltnorth et Diller, 1977; Delvingt, 1978; Stauch, 1981; Eltringham, 1993; Coulibaly et Dibloni, 2007). Il appartient à l'ordre des Ongulés, au sous-ordre des Artiodactyles et à la famille des Hippopotamidés qui comprend deux espèces différentiables par leur taille (Jeannin, 1945):
- l'hippopotame nain (Choeropsis liberiensis, Morton 1844) dont la hauteur au garrot est de $0,90 \mathrm{~m}$ et

l'hippopotame commun (Hippopotamus amphibius, Linné 1758) dont la hauteur au garrot est d'environ $1,40 \mathrm{~m}$.

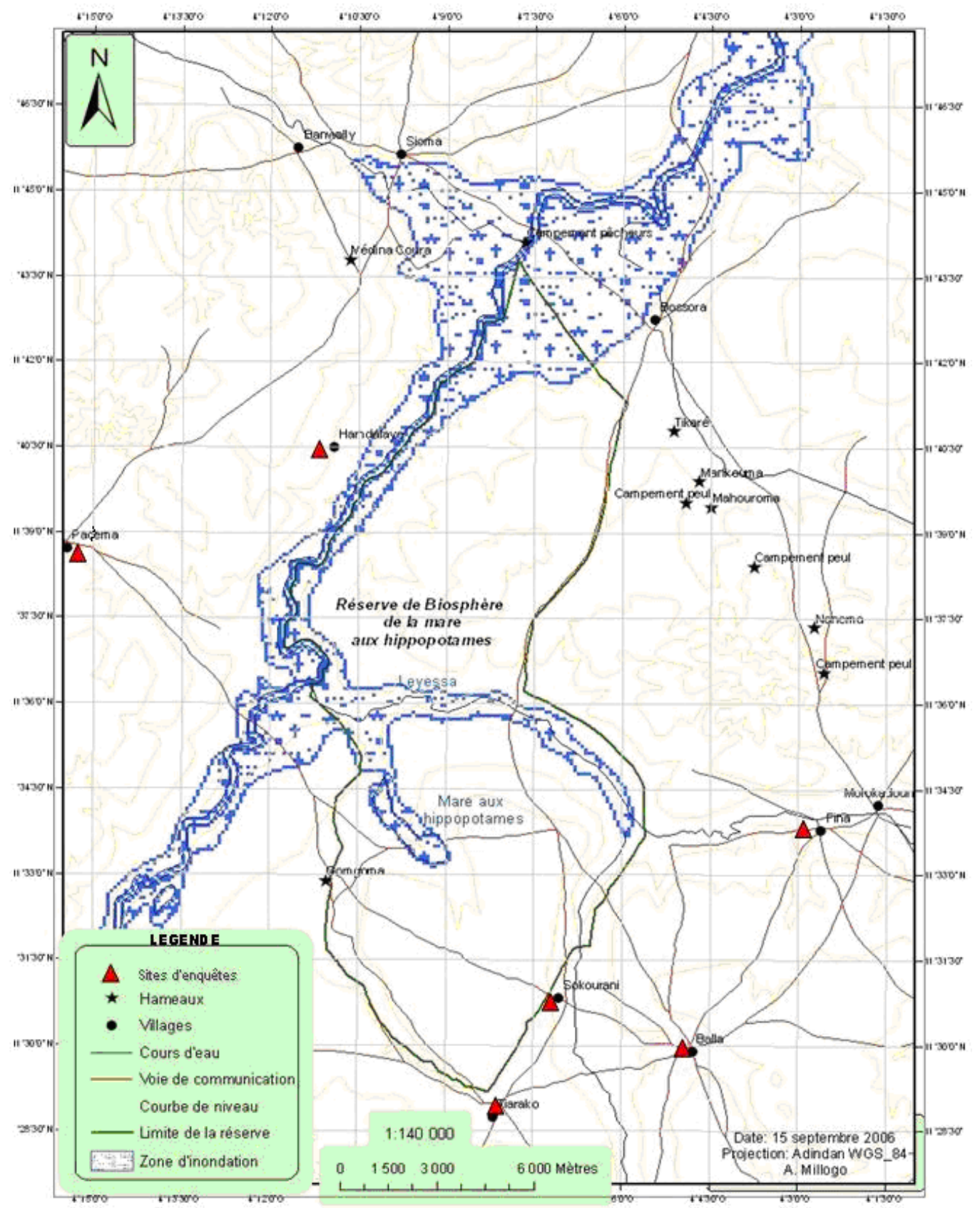

Carte $\mathbf{n}^{\circ}$ 1: Localisation de la Réserve de Biosphère de la Mare aux Hippopotames et des sites d'enquête. 
Ce dernier comprendrait cinq sousespèces difficiles à distinguer sur le terrain (Eltringham, 1993). C'est un herbivore non ruminant, menant une vie grégaire, sédentaire et passant toute la journée dans l'eau. Dans les zones fortement anthropisées, il ne sort de l'eau que les nuits pour rejoindre les gagnages jouxtant son habitat (Haltenorth et Diller, 1977).

Les populations d'hippopotames à travers le continent sont menacées par la disparition de leur habitat, la chasse non contrôlée et les conflits armés. En République Démocratique du Congo (RDC) où la population était estimée à 30.000 individus dans le Parc National du Virunga (Delvingt, 1978), on ne dénombre actuellement que quelques 3000 têtes. Compte tenu de son grand danger d'extinction, il est placé sur la «Liste Rouge » de l'Union Mondiale pour la Nature (UICN, 2006).
Au Burkina Faso, la population de ce gros mammifère amphibie qui était évaluée entre 500 et 600 individus (MECV, 2002), est en régression $\mathrm{du}$ fait de la pression anthropique (culture sur les berges, pêche, braconnage). Il bénéficie de ce fait d'un régime de protection intégrale à la faveur des conventions de Ramsar et de Washington que le Burkina Faso a ratifiées (CONAGESE/ MEE, 1999).

Selon Coulibaly et Dibloni (2007), l'habitat de cet animal est très réduit au Burkina Faso. Il est naturellement présent dans quelques plans d'eau dont les rivières du complexe des parcs W-Arly-Pendjari (WAP), la mare de la Réserve de Biosphère de la Mare aux Hippopotames, les lacs de Bagré et de Tingréla, le fleuve Sourou, les rivières de la Comoé et de la Léraba (Carte 2); il a été introduit dans le parc animalier de Ziniaré à une trentaine de Kilomètres de Ouagadougou

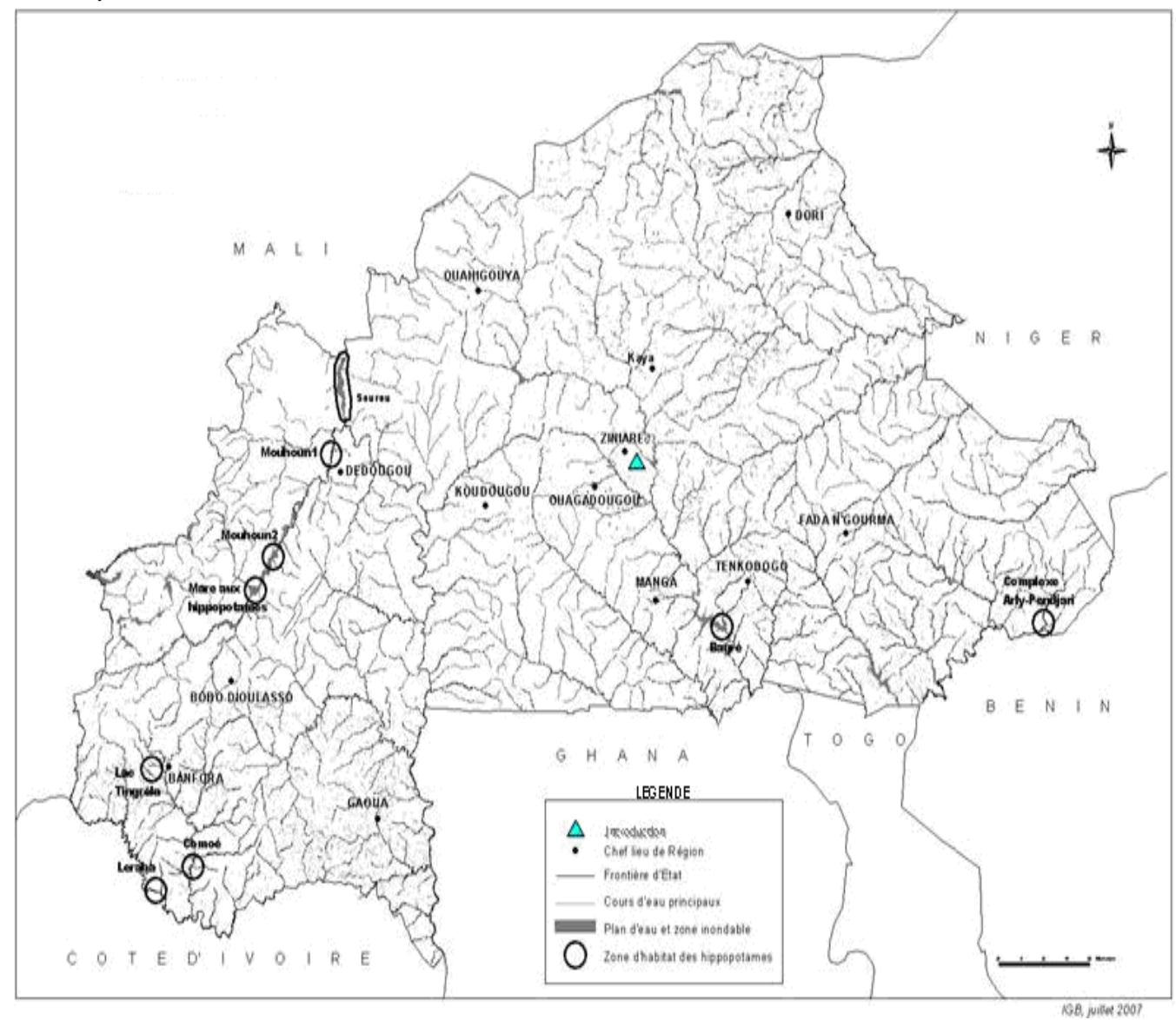

Carte $n^{\circ}$ 2: Distribution de l'habitat des hippopotames au Burkina Faso (Source: Coulibaly et Dibloni, 2007). 


\section{Collecte de données}

La collecte des données s'est effectuée sous forme d'enquêtes formelles dans six des dix villages qui bordent la RBMH et dans les campements des pêcheurs qui sont dans la réserve (Carte1). Le choix de ces villages a été motivé par leur accessibilité et aussi leur proximité avec la mare.

L'enquête réalisée en langue Dioula s'est intéressée aux données relatives à l'effectif et à la migration, à la différenciation sexuelle, au régime alimentaire et aux relations entre les hippopotames et les populations villageoises riveraines à la RBMH.

L'échantillon de notre enquête a concerné les agropasteurs pêcheurs, les anciens chasseurs et tradithérapeutes et les surveillants villageois de la réserve. Ces différents groupes socioprofessionnels nous ont en effet semblés plus proches des hippopotames.

Les données ont été collectées auprès de 77 agropasteurs et pêcheurs composés majoritairement de l'ethnie Bobo (70) et de sept migrants dont cinq de l'ethnie Mossi et deux de l'ethnie Peul; soit au total trois ethnies. Les interviews ont concerné surtout l'ethnie Bobo car les pêcheurs de la Mare étaient constitués surtout de cette ethnie. Ces derniers sont donc plus en contact avec les hippopotames aussi bien dans la mare que dans leurs champs. Les pêcheurs passent toute la saison sèche à la recherche du poisson et se consacrent à l'agriculture pendant l'hivernage. Quant aux migrants, ils sont exclusivement agropasteurs et sont par conséquent tenus à distance de la Mare; ils ne sont en contact avec l'hippopotame que lorsqu'ils voient les empreintes ou les dégâts de celui-ci dans leurs champs pendant l'hivernage.

Malgré le questionnaire guide que nous avons élaboré, les interviews ont été réalisées de manière Semi Structurée suivant la Méthode Accélérée de Recherche Participative (MARP) de Gueye et Freud Emberger (1991). Ces interviews ont été complétées par des observations de terrain. La détermination de certaines espèces végétales a été possible avec la flore illustrée du Sénégal (Berhaut, 1971), les botanistes du laboratoire d'écologie de l'Université de Ouagadougou et de l'herbier national du Burkina Faso basé au
Centre National de Recherche Scientifique et Technologique (CNRST).

\section{RESULTATS}

Effectif et migration des hippopotames selon les populations villageoises

La population enquêtée est constituée de $91 \%$ de l'ethnie Bobo (population autochtone) et $9 \%$ de migrants dont $6,5 \%$ de Mossi et 2,5\% de Peul. L'échantillon d'étude était composé d'anciens chasseurs et de tradithérapeutes $(19,5 \%)$, de pastoralistes $(24,7 \%)$, de pêcheurs $(27,3 \%)$, de surveillants de la réserve $(20,5 \%)$ et autres $(8 \%)$.

La présence des hippopotames dans la réserve a été confirmée par la totalité des personnes enquêtées. Selon $77,6 \%$ des personnes interrogées, l'effectif de ces hippopotames serait d'environ 33 individus vivant entre 2 à 4 troupeaux de tailles variables. Ces personnes ont aussi affirmé qu'il existe une migration saisonnière des hippopotames qui s'effectue au début du mois de juillet, qui est la période au cours de laquelle les hippopotames quittent la mare pour ne revenir qu'au mois d'octobre. Durant leur absence dans la mare, les hippopotames sont présents dans les cours d'eau peu profonds situés aux abords de la réserve.

Critères de différenciation sexuelle des hippopotames selon les populations

Sur l'ensemble des personnes enquêtées (77 au total), 27,3\% n'arrivent pas à faire la différenciation sexuelle chez les hippopotames. Des critères morphologiques et éthologiques ont été cités par $72,7 \%$ des enquêtés (77\% de Bobo et $40 \%$ de Mossi) vivant en bordure de la réserve, qui savaient différencier l'hippopotame mâle de la femelle (Tableau 1). Les migrants Mossi qui distinguent les sexes sont ceux qui font partie de l'équipe de surveillance de la réserve. Selon ces populations riveraines, l'hippopotame mâle différerait de la femelle par une masse plus importante au plan morphologique dont les critères de distinction sont cités suivant les expressions ci-dessous:

- «a bognan ani fari sogo djangnan» pour désigner la grosseur et la longueur du corps;

- «a koungolo bognan» pour parler de la grosseur de la tête ;

- «a ko kolo tamassien» qui désigne l'épine dorsale. 
En ce qui concerne la différenciation sur le plan comportemental, elle serait perceptible lorsque le troupeau est en déplacement. Selon les enquêtés, le mâle supérieur se tiendrait régulièrement en arrière alors que la femelle se place le plus souvent à l'avant du troupeau; enfin la femelle serait souvent aperçue en compagnie des juvéniles.

\section{Régime alimentaire des hippopotames}

Les hippopotames de la Mare prennent leur alimentation dans la mare, les prairies aquatiques et exondées ainsi que dans les champs de cultures pendant l'hivernage. Selon 49,4\% des enquêtés, les hippopotames vont paître la nuit dans les gagnages situés sur les berges de la mare entre $19 \mathrm{~h}$ et $20 \mathrm{~h}$ et retournent dans l'eau au petit matin (entre $5 \mathrm{~h}$ et $6 \mathrm{~h})$.

Selon les agropêcheurs et les surveillants de la réserve, le régime alimentaire de l'hippopotame serait constitué préférentiellement par des herbacées $(53,3 \%$ des citations), suivies par des feuilles d'arbustes $(24,3 \%)$ et le cas échéant par les rejets et les plantes céréalières $(22,4 \%)$. Les prairies aquatiques et les champs des cultures céréalières (riz, Oryza barthii A. Chev. ; maïs, Zea mays L.) situées près des berges seraient les principales aires de pâture des hippopotames (Photo 1 et 2) pendant la saison des pluies. Une quarantaine d'espèces végétales ont été identifiées par les agropêcheurs (Tableau 2) dont les principales espèces appétées sont des herbacées connues sous le nom de «mali biin » en langue Dioula. Il s'agirait de: Leersia hexandra Swartz, Echinochloa stagnina P. Beauv., Echinochloa colona Link, Schizachyrium sanguineum (Retz.) Alston, Andropogon pseudapricus Stapf, Cissampelos mucronata A. Rich, Sporobolus pyramidalis P. Beauv.et Digitaria horizontalis Willd.

Relations hippopotames et populations villageoises riveraines de la RBMH

L'enquête réalisée a permis de déterminer les avantages et les conflits issus de la cohabitation hommes hippopotames

Tableau 1: Critères de diagnose chez les hippopotames selon les populations riveraines de la RBMH.

\begin{tabular}{|c|c|c|c|c|}
\hline Critères & & Mâle & Femelle & $\begin{array}{c}\text { Fréquences de } \\
\text { citation }(\%)\end{array}$ \\
\hline \multirow{6}{*}{ Morphologique } & Tête & Épaisse & Mince & 69,2 \\
\hline & Abdomen & Loin du sol & Près du sol & 23,1 \\
\hline & Corps & Long et gros & Trapue (ramassée) & 37,5 \\
\hline & Canines & Grosses & Minces & 12,8 \\
\hline & Epine dorsale & Saillante & Effacée & 34,0 \\
\hline & $\mathrm{Nez}$ & Plat & Droit & 7,7 \\
\hline \multirow{3}{*}{ Ethologique } & Déplacement & Dernier du troupeau & En tête du troupeau & 53,4 \\
\hline & Soins parentaux & Non accompagné & $\begin{array}{l}\text { Souvent accompagné } \\
\text { d'un jeune }\end{array}$ & 5,4 \\
\hline & $\begin{array}{l}\text { Réaction face à un } \\
\text { danger }\end{array}$ & Premier à réagir & Lent à réagir & 12,2 \\
\hline
\end{tabular}

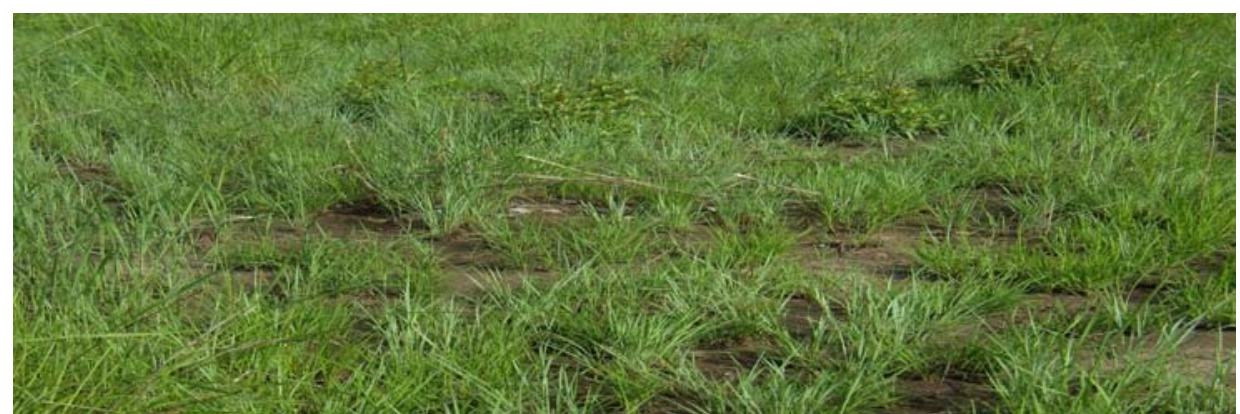

(Photo DIBLONI, Juin 2008)

Photo n'1: Prairie aquatique à Schizachyrium sanguineum (Retz.) Alston. 


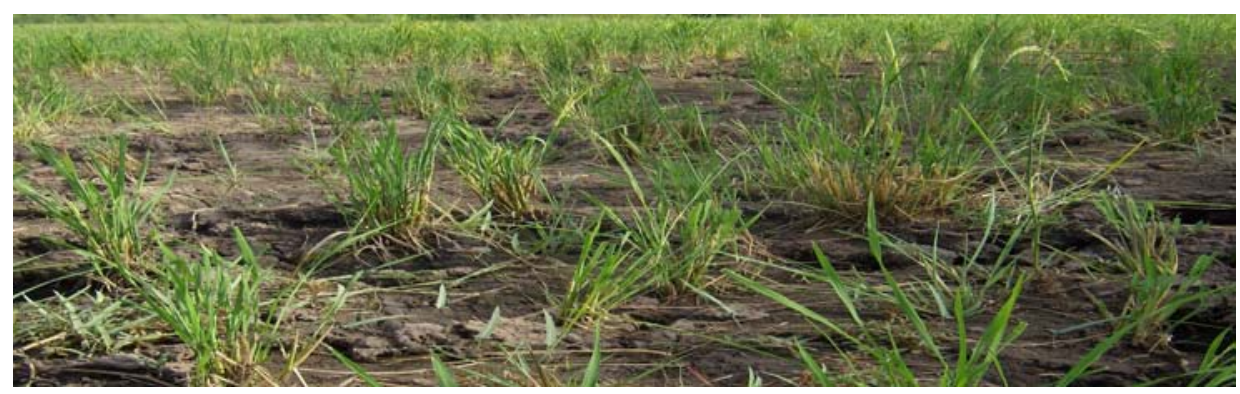

(Photo DIBLONI, Octobre 2007)

Photo $\mathbf{n}^{\circ} 2$ : Périmètre rizicole, à proximité des rives du fleuve Mouhoun, ravagé par un troupeau d'hippopotames.

ainsi que la place de ces hippopotames dans la tradition des sociétés villageoises riveraines. S'agissant des conflits, les hippopotames seraient responsables de dégâts sur les cultures et les filets de pêche (soit respectivement $49,5 \%$ et $28,6 \%$ de citation) ainsi que de quelques accidents mortels sur les pêcheurs par des femelles suitées (figure 1).

A cộté de ces rapports conflictuels, la présence des hippopotames dans la Mare de la Réserve de Biosphère aurait des effets bénéfiques en terme d'écotourisme (80,5\% des citations), de génération de revenus par le tourisme et d'accroissement de la production piscicole $(76,6 \%$ de citations), d'accroissement de biodiversité animale $(43,3 \%$ des citations) et d'éducation des enfants $(30,6 \%$ des citations). Il existe d'autres domaines non moins importants comme la pharmacopée et les rites culturels reconnus par $10 \%$ des personnes enquêtées (figure 2). Sur le plan pharmacologique, la peau de l'hippopotame soignerait des démangeaisons. Les os de la queue et du front seraient recherchés pour invoquer des puissances occultes. S'agissant des rites culturels, la viande de l'hippopotame et à défaut du crocodile serait exigée avant la programmation de certaines cérémonies coutumières annuelles. Selon les populations $\mathrm{du}$ village de Sokourani (Bobo), lorsqu'un hippopotame tuait un homme et que ce dernier restait immergé au fond de l'eau, il fallait procéder à certains sacrifices pour permettre au cadavre de remonter en surface. Aussi, lorsqu'un hippopotame mourait dans l'eau il fallait recourir à des sacrifices pour demander la clémence des ancêtres avant d'accéder à nouveau à la mare; ce n'est qu'ensuite que la viande était distribuée aux populations villageoises riveraines pour consommation. Les populations villageoises de Sokourani affirment en outre qu'en traversant les endroits de la mare ou étaient immergés les hippopotames, il suffisait de prononcer qu'il y a «un bois» en dessous et la traversée s'effectuait librement sur le dos de ces animaux.

\section{DISCUSSION}

Les environs de la Réserve de Biosphère de la Mare aux Hippopotames constituent une zone d'accueil pour de nombreux migrants du Nord du Burkina Faso et de ce fait regroupent une population très hétérogène ayant des connaissances assez imparfaites sur la réserve et ses potentialités. Par ailleurs, la technique d'échantillonnage des villages riverains et des personnes interviewées (77) peut ne pas être suffisamment représentative de la diversité socioculturelle de la zone ou de l'origine ou statut des enquêtés. Malgré ces limites, les résultats suggèrent que l'hippopotame, en tant qu'animal emblématique de cette Réserve de Biosphère, est bien connu de la majorité des populations riveraines qui arrivent à faire la différenciation sexuelle chez cet animal, à identifier son régime alimentaire et à lui reconnaître des valeurs culturelles et socioéconomiques.

La taille du troupeau d'hippopotames peuplant la Mare de la Réserve de Biosphère a peu varié au cours des deux dernières décennies. L'effectif rapporté par les riverains, particulièrement la communauté des pêcheurs, n'est guère différente des effectifs fournis par les travaux de l'ENGREF (1989) et de Poussy et Bationo (1991) qui étaient respectivement de 39 et 35 individus. L'inventaire de l'UCF/Haut bassin (2004) corrobore le chiffre des pêcheurs. Ces derniers constituent donc une source d'information 
Tableau 2 : Espèces végétales appétées par les hippopotames selon Bobo des environs de la RBMH.

\begin{tabular}{|c|c|c|}
\hline Effectif & Famille & Espèces \\
\hline 1 & Araceae & Pistia stratiotes L. \\
\hline 2 & Asteraceae & Grangea maderaspatana (L.) Poir. \\
\hline 3 & & Ambrosia senegalensis \\
\hline 4 & Azolaceae & Azolla africana Desv. \\
\hline 5 & Capparidaceae & Crataeva adansonii DC. \\
\hline 6 & Ceratophyllaceae & Ceratophyllum demersum $\mathrm{L}$. \\
\hline 7 & Caesalpiniaceae & Cassia mimosoides \\
\hline 8 & Convolvulaceae & Merremia tridenta (L.) Hallier f. \\
\hline 9 & & Ipomea aquatica Forsk. \\
\hline 10 & Cyperaceae & Cyperus digitatus Roxb. \\
\hline 11 & & Cyperus rotondus $\mathrm{L}$. \\
\hline 12 & Euphorbiaceae & Phyllanhtus muellerianus (O. K. Tze)Exell. \\
\hline 13 & Fabaceae & Sesbania sesban (Linn.) Merrill. \\
\hline 14 & Ficoïdeae & Oldenlandia $\mathrm{sp}$ \\
\hline 15 & Menispermaceae & Cissampelos mucronata A. Rich.* \\
\hline 16 & Mimosaceae & Mimosa pigra $\mathrm{L}$. \\
\hline 17 & & Neptunia oleracea Lour. \\
\hline 18 & Nympheaceae & Nymphea lotus L. \\
\hline 19 & Onagraceae & Ludwigia adscendans $\mathrm{P}$ \\
\hline 20 & & Andropogon ascinodis C. B. Cl. \\
\hline 21 & & Andropogon pseudapricus Stapf* \\
\hline 22 & & Digitaria horizontalis Willd.* \\
\hline 23 & & Echinochloa colona Link.* \\
\hline 24 & & Echinochloa stagnina P. Beauv. \\
\hline 25 & Poaceae & Leersia hexandra Swartz* \\
\hline 26 & & Monocymbium ceresiiforme (Nees) Stapf. \\
\hline 27 & & Oryza barthii A. Chev. \\
\hline 28 & & Paspalum orbiculare (Presl.) \\
\hline 29 & & Schizachyrium sanguineum (Retz.) Alston* \\
\hline 30 & & Sporobolus pyramidalis P. Beauv.* \\
\hline 31 & & Vetiveria nigritana Stapf \\
\hline 32 & & Zea mays $\mathrm{L}$. \\
\hline 33 & Polygonaceae & Polygonum limbatum Meins. \\
\hline 34 & & Polygonum senegalensis Meins. \\
\hline 35 & Rubiaceae & Morelia senegalensis A. Rich. \\
\hline 36 & & Mitragyna inermis (Wild) Kuntze \\
\hline 37 & Sapindaceae & Cardiospermum halicacabum L. \\
\hline 38 & Sterculiaceae & Melochia corchorifolia L. \\
\hline 39 & Trapaceae & Trapa natans L. \\
\hline 40 & Verbenaceae & Stachystarpheta angustifolia (Mill.) \\
\hline
\end{tabular}




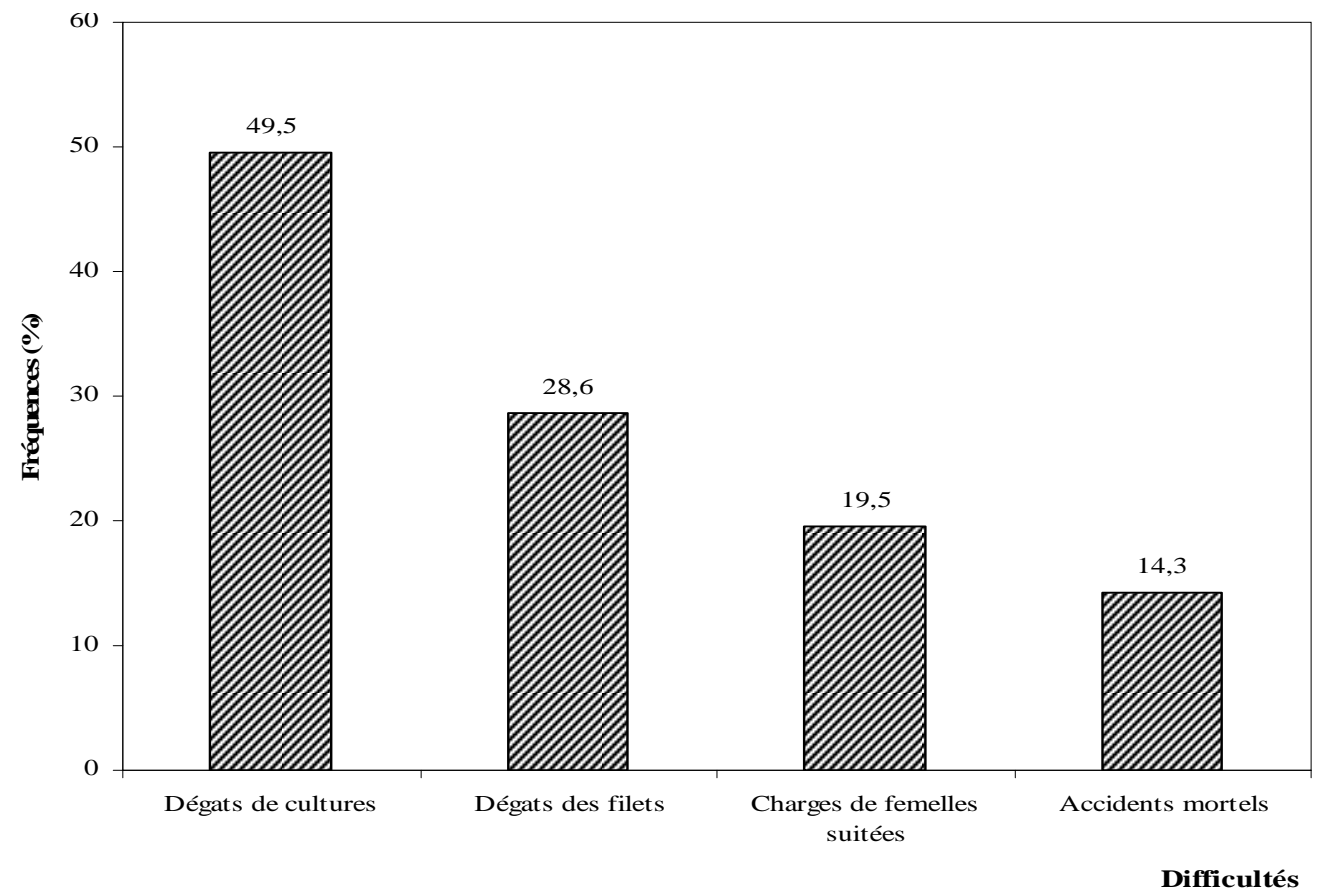

Figure 1: Fréquence (\%) de citation des difficultés dues aux hippopotames.

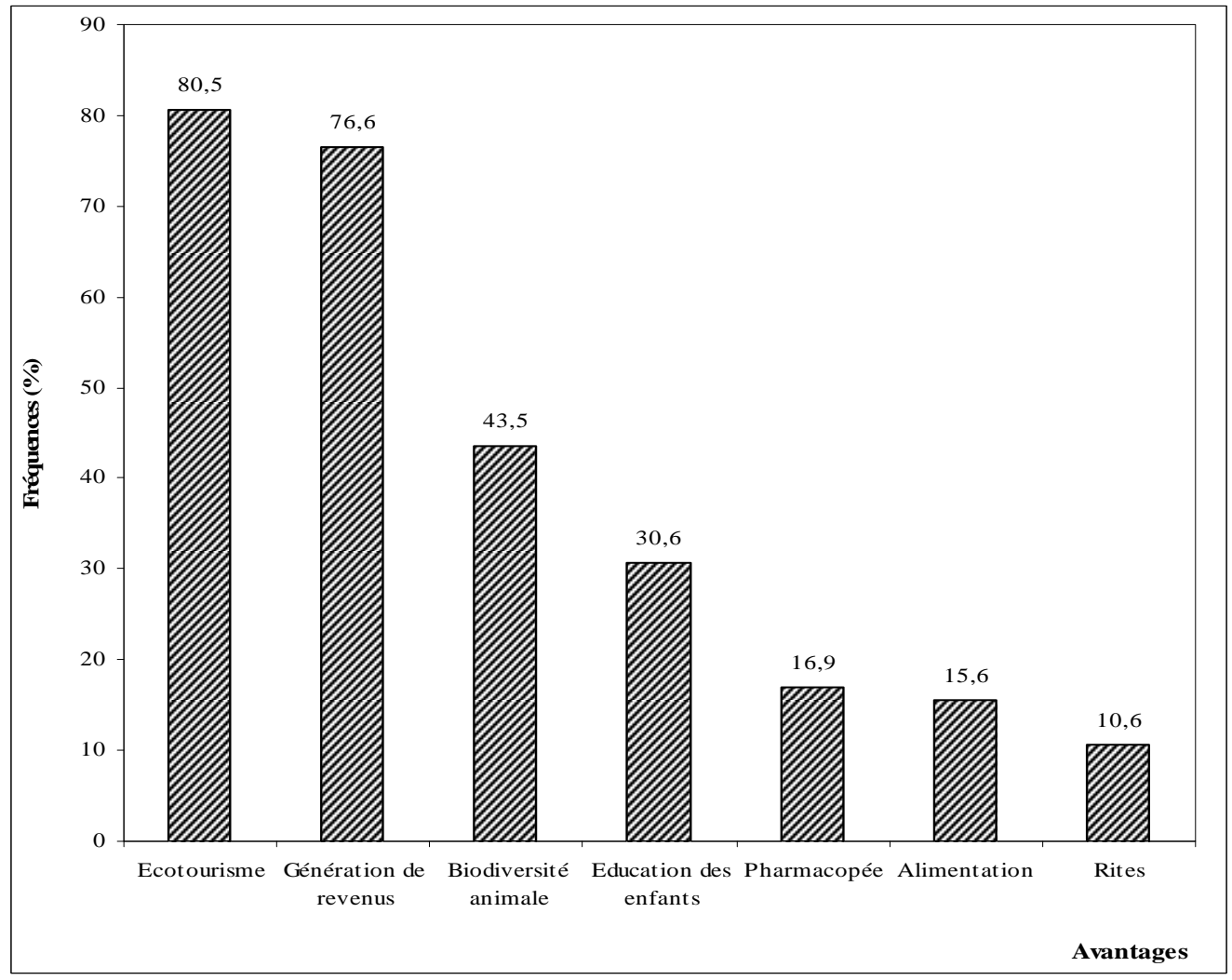

Figure 2: Fréquence $(\%)$ de citation des avantages des hippopotames. 
précieuse en cas d'absence d'inventaire récent.

La relative stabilité de l'effectif des hippopotames de cette mare pourrait être expliquée par deux hypothèses majeures: le braconnage de ces animaux pendant leur migration de juillet à septembre ou le non retour à la RBMH de certains individus qui poursuivent leur trajet dans le Mouhoun puis le Sourou, deux plans d'eau où leur présence a été signalée (Coulibaly et Dibloni, 2007).

Les différentiations morphologique et éthologique entre les hippopotames mâles et femelles, décrites par les populations villageoises riveraines, semblent être en adéquation avec les études antérieures. Sur le plan morphologique les mensurations effectuées par plusieurs auteurs montrent que l'hippopotame mâle a un poids et une taille supérieurs à ceux de la femelle (Kingdom, 1997; Eltringham, 1999; Lamarque, 2004). Cette différence pourrait s'expliquer par le fait que l'hippopotame femelle atteint son poids maximum en moyenne à 25 ans alors que l'hippopotame mâle semble ne jamais arrêter sa croissance (Eltringham, 1999). De même, pour ce qui concerne le comportement des hippopotames en déplacement décrits par les enquêtées, Eltringham (1999) rapporte que les groupes d'hippopotames sont souvent composés de femelles accompagnées de leurs petits sous l'autorité territoriale d'un mâle dominant.

Le rôle et l'importance de la flore herbacée dans le régime alimentaire de l'hippopotame rapportés précédemment par BERD (2004), Noirard et al. (2004) et Kabré et al. (2006) sont ici confirmés par les observations de riverains et utilisateurs de la mare. Les préférences alimentaires des hippopotames portaient sur les herbacées sauvages ou cultivées (riz, maïs) et accessoirement sur les parties foliaires des espèces ligneuses.

Parmi les espèces appétées par les hippopotames selon les enquêtées, six d'entre elles ont été identifiées dans des fèces d'hippopotames par Noirard et al. (2004) et Kabré et al. (2006). Il s'agit de Echinochloa stagnina P. Beauv., Echinochloa colona Link, Paspalum orbiculare (Presl.), Andropogon pseudapricus Stapf, Oryza barthii A. Chev. et de Cyperus sp.
La proximité des cultures céréalières sur les berges constitue une source de conflits entre intérêts agricoles et présence des hippopotames. Ces types de conflits sont surtout fréquents en saison pluvieuse dans les exploitations agricoles situées à la lisière des réserves de faune par les grands mammifères sauvages (Okoumassou et al., 2004; Binot et al., 2006).

A coté de sa contribution socioéconomique (génération des revenus par l'écotourisme et l'accroissement de la production piscicole) qui est la plus importante et la mieux connue des riverains, l'hippopotame de la Mare présente également une valeur socioculturelle pour une faible proportion de la population $(10 \%$ des enquêtés), sans doute les initiés ou les autochtones séculaires des villages riverains.

L'utilisation de l'hippopotame (ou de sa viande) dans les rites ancestraux de la localité est considérée comme une question tabou et ceci en regard des mesures de protection intégrale dont jouit cet animal et du respect des secrets des coutumes. Les usages socioculturels des animaux sauvages sont encore d'actualité pour les populations rurales du Burkina Faso (Coulibaly, 2006) et pour le cas spécifique de l'hippopotame, seulement les personnes d'un certain âge et d'une position sociale donnée pouvaient en parler librement.

S'agissant du partage d'un hippopotame mort au sein des communautés riveraines, selon Larénie et Huet (2006), cette pratique existerait également chez les Bijagos en Guinée-Bissau et répondrait au fait que l'hippopotame déchire les hommes qu'il tue.

La bonne connaissance des effectifs, du sexe et du régime alimentaire de l'hippopotame par les populations locales autant que son importance symbolique pour ces dernières plaide pour une plus grande implication de leur part dans sa gestion. Ainsi, l'action de l'AGEREF qui consiste en la surveillance de la réserve doit être poursuivie et les membres formés doivent être dotés de matériels adéquats pour les patrouilles. Il est important de rappeler qu'à l'étape actuelle où l'Etat Burkinabé vient d'ériger tout le territoire national en communes, il est opportun de redéfinir les rôles et les compétences de tous les acteurs de cette Réserve de la Biosphère afin de favoriser une 
gestion plus concertée au profit des différentes communautés.

Pour conclure, l'enquête réalisée, auprès des populations riveraines de la RBMH, a montré que celles-ci ont acquis au fil du temps une bonne connaissance de la faune sauvage en général et de l'hippopotame en particulier. Elles connaissent l'hippopotame dans sa morphologie et son comportement; ce qui les conduit à faire la diagnose sexuelle sur des bases anatomiques. Les connaissances endogènes portent également sur la dynamique de population, le régime alimentaire et les valeurs socioéconomique et culturelle de cet animal emblématique de la réserve. Compte tenu de toutes les connaissances dont disposent les populations riveraines, une gestion concertée de la réserve et de ses environs devrait se baser sur une collaboration étroite entre usagers producteurs, associations civiles (AGEREF), l'administration communale, les partenaires scientifiques et les services techniques chargés de la conservation.

\section{REMERCIEMENTS}

Les auteurs tiennent à remercier vivement le GEF-MAB/UNESCO pour le financement de cette étude, Messieurs Alfred N. MILlOGO (Ecologue du PAGEN) et Oumar KABORE (CTIG/INERA) ainsi que les populations des villages riverains de la Réserve de la Biosphère de la Mare aux Hippopotames du Burkina Faso, pour leur précieuse collaboration.

\section{REFERENCES BIBLIOGRAPHIQUES}

Bélem OM. 2008. Les galeries forestières de la Réserve de Biosphère de la Mare aux Hippopotames du Burkina Faso: caractéristiques, dynamique et ethnobotanique. Thèse de doctorat ès Sc. Nat. Université de Ouagadougou, 248.

BERD. 2004. Diagnostic des ressources en eau de la mare de la Réserve de Biosphère de la Mare aux Hippopotames. Rapport provisoire, PAGEN/UCF-Hauts Bassins, Burkina Faso.

Binot A, Castel V, Caron A. 2006. L'interface faune-bétail en Afrique subsaharienne. Sécheresse, 17(1-2): 349-361.

Bouché P. 2005. Inventaire aérien des populations de grande faune dans les sites du PAGEN: Forêt Classée et Réserve partielle de faune de Comoé-Léraba; Forêts classées de Boulon et de Koflandé, Réserve de Biosphère de la Mare aux Hippopotames; Parc National Kaboré Tambi, (Version provisoire) PAGEN.

Chardonnet P. 1995. Faune Sauvage Africaine: La Ressource Oubliée. Tome II, CEE; 288p.

CICRED. 2006. Mobilité spatiale de la population : nécessité de développement et de risques de dégradation de l'environnement dans l'Est et le SudOuest du Burkina Faso. Rapport final, CONVENTION PRIPODE, CICREDINSS BF5.

CONAGESE. 1999. Monographie nationale sur la diversité biologique du Burkina Faso. MEE.

Coulibaly ND. 2006. Valeurs socioculturelles $\mathrm{du}$ poisson: Thérapie et mysticisme. Carrefour Africain, 1128:18

Coulibaly ND, Dibloni OT. 2007. Faune sauvage: à la découverte de l'hippopotame commun. Notre Environnement, 37:18-19.

Delvingt W. 1978. Ecologie de l'hippopotame (Hippopotamus amphibius L.) au Parc National de Virunga. Thèse de Doctorat, FUSAGx, Belgique, 334.

ENGREF. 1989. Réserve de Biosphère de la Mare aux Hippopotames. Etude préalable à un aménagement de la réserve et de sa zone périphérique. UNESCO/MAB, MEE, Burkina Faso.

Haltenorth T, Diller H. 1977. Mammifères d'Afrique et de Madagascar. Delachaux et Niestlé.

Eltringham SK. 1993. The Common Hippopotamus (Hippopotamus amphibious). In Pigs, Peccaries and Hippos: Status Survey and Action Plan, Olivier WLR (ed). Gland Switzerland IUCN; 161-171.

Eltringham SK. 1999. The Hippos: Natural History and Conservation. Academic Press: London.

Gomgnimbou M, Bonou GB. 1996. Etude socio-économique de la situation migratoire des villages riverains de la Réserve de Biosphère de la Mare aux Hippopotames. Rapport atelier DREEFHB/INERA, 20-21 octobre 1997, BoboDioulasso. 
Huklop P. 2000. Elephant crop raiding patterns in areas around Kibal National Park (KNP), Uganda. In Human-Wildlife Conflict: Identifying the Problem and Possible Solutions (Albertine Rift Technical Report Series), Hill C, Osborn F, Plumptre AJ (ed). Wildlife Conservation Society; (1):107-115.

Jeannin A. 1945. Les Bêtes de Chasse de l'Afrique Française. Payot: Paris.

Gueye B, Freud Emberger HS. 1991. Introduction à la MARP (Rapid Rural Appraisal): Quelques notes pour appuyer une formation pratique. London.

Kabré TA, Koné L, Saley H, Nandnaba S, Sawadogo BB. 2006. Rythme circadien et régime alimentaire de l'hippopotame amphibie dans les bassins de la Volta et de la Comoé. Sciences et Techniques, Sciences Naturelles et Agronomie, 28(1 et 2): 73-88.

Kièma S. 2007. Elevage extensif et conservation de la diversité biologique dans les aires protégées de l'Ouest burkinabé. Arrêt sur leur histoire, épreuves de la gestion actuelle, état et dynamique de la végétation. Thèse de doctorat, Université d'Orléans, France, 562.

Kingdom J. 1997. The Kingdom Field Guide to African Mammals. Academic Press: London.

Lamarque F. 2004. Les grands mammifères du complexe WAP. UE, CIRAD, ECOPAS.

MECV. 2006. Programme cadre de gestion durable des ressources forestières et fauniques au Burkina Faso (2006-2015) : composante gestion de la faune et des aires de protection fauniques. Burkina Faso.

Noirard C, Le Berre M, Ramousse R, Sépulcre C, Joly P. 2004. Diets of sympatric Hippopotamus (Hippopotamus amphibius) and Zebus (Bos indicus) during the dry season in the $\langle\mathrm{W} »$ National Park (Niger Republic). Game and Wildl. Sci., 21(3): 423-431.

Okoumassou K, Durlot S, Akpamou K,
Segniagbeto H. 2004. Impacts humains sur les aires de distribution et couloirs de migration des éléphants du Togo. Pachyderm, 36: 69-79.

Poda JN. 1997. Le Programme de l'Homme et la Biosphère (MAB) et la Réserve de Biosphère de la Mare aux Hippopotames du Burkina Faso: Etat des lieux et perspectives de renforcement. Document de travail. CNRST, Burkina Faso.

Poussy M, Bakyono E. 1991. Aménagement de l'habitat de l'hippopotame. Mare aux hippopotames. Rapport d'exécution du Projet UNESCO/BREDA-IRBET/CNRST, Ouagadougou.

Sam MK, Haziel C, AK, Barnes RFW. 2002. Crop damage by elephants in the red volta area during the 1997 harvesting season. In Human-Wildlife Conflict: Identifying the Problem and Possible Solutions (Albertine Rift Technical Report Series), Hill C, Osborn F, Plumptre AJ (eds). Wildlife Conservation Society; (1):127-136

Stauch A. 1981. Mammifères. In Flore et Faune Aquatiques de l'Afrique SaheloSoudanienne (Tome 2), Durand JR, Lévêque C (eds). ORSTOM-IDT: Paris; (45) : 841-847.

Taïta P. 1997. Contribution à l'étude de la flore et de la végétation de la Réserve de Biosphère de la Mare aux Hippopotames (Bala, Ouest du Burkina Faso). Thèse de doctorat de troisième cycle, Université de Ouagadougou, p. 137.

UCF/Haut Bassin. 2004. Etude d'inventaire de la population d'Hippopotamus amphibius de la Réserve de Biosphère de la Mare aux Hippopotames. PAGEN/MECV, Burkina Faso.

UICN. 2006. Liste rouge de l'UICN des espèces menacées. Disponible au « http://www.developpement-durablelavenir.com/2006/05/03/liste-rouge-de-1iucn-des-especes-menacees-2006 » consulté le 30 janvier 2007.

Vermeulen C. 2001. Aires protégées et accroissement démographique. Canopée, 20: $10-12$. 